\title{
Study of the applicability of the curlometer technique with the four Cluster spacecraft in regions close to Earth
}

\author{
S. Grimald ${ }^{1,2}$, I. Dandouras ${ }^{1,2}$, P. Robert ${ }^{3}$, and E. Lucek ${ }^{4}$ \\ ${ }^{1}$ Université de Toulouse, UPS-OMP, UMR5277, Institut de Recherche en Astrophysique et Planétologie, Toulouse, France \\ ${ }^{2}$ CNRS, Institut de Recherche en Astrophysique et Planétologie, Toulouse, France \\ ${ }^{3}$ Laboratoire de Physique des Plasmas, CNRS, Paris, France \\ ${ }^{4}$ Blackett Laboratory, Imperial College, London, UK \\ Correspondence to: S. Grimald (grimald@cesr.fr)
}

Received: 24 June 2011 - Revised: 14 February 2012 - Accepted: 17 February 2012 - Published: 27 March 2012

\begin{abstract}
Knowledge of the inner magnetospheric current system (intensity, boundaries, evolution) is one of the key elements for the understanding of the whole magnetospheric current system. In particular, the calculation of the current density and the study of the changes in the ring current is an active field of research as it is a good proxy for the magnetic activity. The curlometer technique allows the current density to be calculated from the magnetic field measured at four different positions inside a given current sheet using the Maxwell-Ampere's law. In 2009, the CLUSTER perigee pass was located at about $2 R_{\mathrm{E}}$ allowing a study of the ring current deep inside the inner magnetosphere, where the pressure gradient is expected to invert direction. In this paper, we use the curlometer in such an orbit. As the method has never been used so deep inside the inner magnetosphere, this study is a test of the curlometer in a part of the magnetosphere where the magnetic field is very high (about $4000 \mathrm{nT}$ ) and changes over small distances $(\Delta B=1 \mathrm{nT}$ in $1000 \mathrm{~km})$. To do so, the curlometer has been applied to calculate the current density from measured and modelled magnetic fields and for different sizes of the tetrahedron. The results show that the current density cannot be calculated using the curlometer technique at low altitude perigee passes, but that the method may be accurate in a [ $3 R_{\mathrm{E}} ; 5 R_{\mathrm{E}}$ ] or a [ $6 R_{\mathrm{E}} ; 8.3 R_{\mathrm{E}}$ ] $\mathrm{L}$-shell range. It also demonstrates that the parameters used to estimate the accuracy of the method are necessary, but not sufficient conditions.
\end{abstract}

Keywords. Magnetospheric physics (Current systems)

\section{Introduction}

The existence of a current circling the Earth in the near Earth region was first suggested by Singer (1957). The ring current can be visualized as a toroïdal ring current flowing around the Earth at geocentric distances from $2 R_{\mathrm{E}}$ to $9 R_{\mathrm{E}}$ (where $R_{\mathrm{E}}$ is the Earth radius). Two phenomena are responsible for the existence of a current at this position: first, the gradient drift of particles above about $1 \mathrm{keV}$ which produces a westward current and, second, the magnetisation current whose direction depends on the direction of the particle pressure gradient. Studies of the particle pressure in the inner magnetosphere show that the direction of its gradient changes when travelling in the ring current: it is directed outwards close to the Earth and in the opposite direction (inward) far from it (Lui et al., 1987, Lui and Hamilton, 1992). These observations indicate that two ring currents exist: an eastward one, below a given altitude, and a westward one, above it. This effect had been observed in satellite data (see Le et al., 2004; Jorgensen et al., 2004) and the current reversal boundary had been located between $3 R_{\mathrm{E}}$ and $4 R_{\mathrm{E}}$.

The ring current is very sensitive to the variations of the magnetic activity. Magnetic storms impact the position of its boundaries (Zaharia et al., 2006; Chen et al., 2006), its intensity (Hamilton et al., 1988; Smith and Hoffman, 1973; Jordanova, 2003; Jordanova et al., 2006), its composition (Gloeckler et al., 1985; Krimigis et al., 1985; Hamilton et al., 1988; Daglis et al., 1993) and are responsible for the observation of nose-like structures (Smith and Hoffman, 1973; Ejiri, 1978; Shirai et al., 1997; Vallat et al., 2007; Dandouras et al., 2009) or energy bands (Vallat et al., 2007) in the 


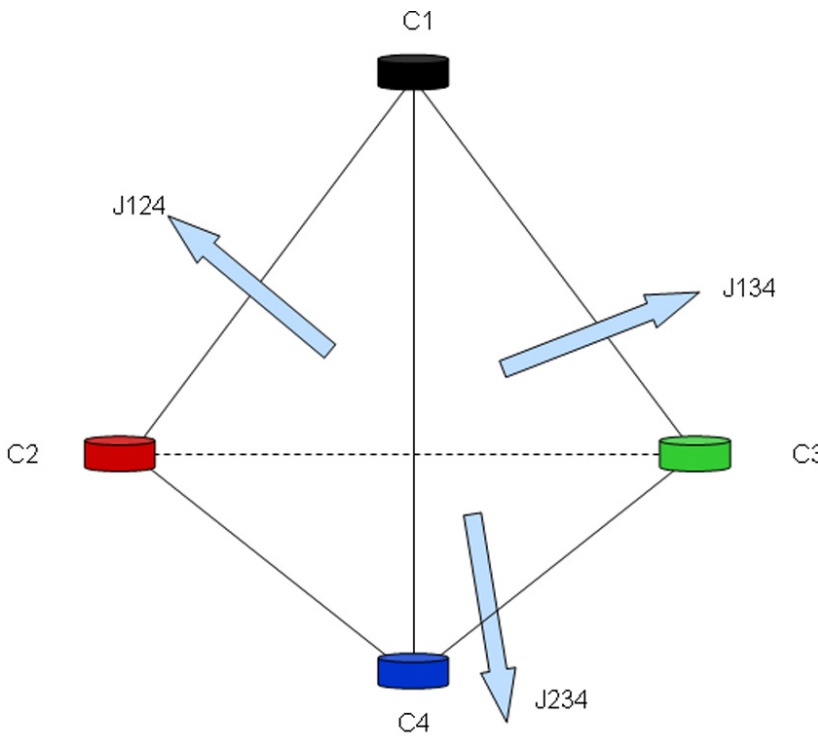

Fig. 1. Illustration of the average current density estimation using the curlometer (adapted from Dunlop et al., 1988).

energy/time spectrograms of the particles. It is well known that the ring current evolution depends, first, on the particle injections during increasing of the magnetic activity and, second, on the loss mechanisms (Daglis et al., 1999), but the current driven by this population as well as the position of the reversal boundary remain to be determined. One way to find it is to estimate the current density.

Two methods have been developed in order to calculate the current density: the method of the pressure gradient, which uses the plasma fluid momentum equation (Lui et al., 1987; Roelof et al., 2004), and the curlometer technique, which uses the Maxwell-Ampere law (Robert and Roux, 1993; Robert et al., 1998a; Dunlop et al., 2002; Dunlop and Balogh, 1993). The first method requires the determination of pressure gradients, which is quite difficult using satellite data. The second method requires magnetic field measurements at different points inside the current sheet. The four satellites of the Cluster mission cross the ring current at different positions in their orbits. Their magnetic field data have been used by Vallat et al. (2005) to calculate the current density for different events observed in 2002, when the Cluster perigee was located at $4 R_{\mathrm{E}}$. This position of the perigee pass allowed the study of the westward ring current, the partial one and the inner plasmasheet, which brought new results about the latitudinal extent of the ring current and the dependence of its intensity on the magnetic activity in the evening sector. Since 2009, the Cluster perigee altitude has decreased to about $2 R_{\mathrm{E}}$. As a consequence, the orbit crosses the region where the current reversal boundary, as defined by Le at al. (2004) and by Jorgensen et al. (2004), is expected. A study of the current density along such an orbit could give us a better knowledge of the current density deeper inside the inner magnetosphere than ever calculated before, but could also provide a more precise location of the reversal boundary. Here, we use the curlometer technique to study the ring current for ring current crossings observed in 2009. As the curlometer has never been used so deep inside the inner magnetosphere, this study is also a test of the curlometer in a part of the magnetosphere where the magnetic field is very high (about $4000 \mathrm{nT}$ ) and varies over small distances $(\Delta B=1 \mathrm{nT}$ in $1000 \mathrm{~km})$. Section 2 presents briefly the curlometer technique. Section 3 presents the 15 May 2009 ring current crossing and the results from the curlometer for this event. Sections 4 and 5 present a test of the curlometer along the 15 May 2009 orbit and for different separations. Conclusions are given in Sect. 6.

\section{The curlometer technique}

The curlometer technique has been described by Dunlop et al. (1988, 2002), Chanteur and Mottez (1993) and Robert et al. (1998a). It uses the Maxwell-Ampere's law to estimate the current density $\boldsymbol{J}$ through the tetrahedron formed by four spacecraft. This section gives a quick description of the method.

\subsection{Basic definitions}

Assuming stationarity in the studied medium, the MaxwellAmpere's law can be written as:

$\mu_{0} \boldsymbol{J}=\operatorname{curl} \boldsymbol{B}$

Using four spacecraft travelling together in a tetrahedral configuration (see Fig. 1), it is possible to determine the average current density normal to each face of the tetrahedron. Assuming the current density is a constant in the whole surface and the magnetic field changes very slowly, relation (1) can be written as (Dunlop et al., 1988):

$\mu_{0} \boldsymbol{J}_{i j k} \cdot\left(\Delta \boldsymbol{r}_{i k} \times \Delta \boldsymbol{r}_{j k}\right)=\Delta \boldsymbol{B}_{i k} \cdot \Delta \boldsymbol{r}_{j k}-\Delta \boldsymbol{B}_{j k} \cdot \Delta \boldsymbol{r}_{i k}$

Where $i, j$ and $k$ refer to the satellite number, $\boldsymbol{J}_{i j k}$ is the average current density normal to the surface made by the satellites $i, j$ and $k, \Delta \boldsymbol{r}_{i k}=\boldsymbol{r}_{i}-\boldsymbol{r}_{k}$ is the distance between the satellite $i$ and the satellite $j$, and $\Delta \boldsymbol{B}_{i k}=\boldsymbol{B}_{i}-\boldsymbol{B}_{k}$ is the magnetic field difference between the satellite $i$ and the satellite $j$. As an example, $\boldsymbol{J}_{123}$ is the average current density normal to the surface formed by $\mathrm{C} 1, \mathrm{C} 2$ and $\mathrm{C} 3$ (see Fig. 1). Using relation (2):

$\mu_{0} \boldsymbol{J}_{123} \cdot\left(\Delta \boldsymbol{r}_{13} \times \Delta \boldsymbol{r}_{23}\right)=\Delta \boldsymbol{B}_{13} . \Delta \boldsymbol{r}_{23}-\Delta \boldsymbol{B}_{23} . \Delta \boldsymbol{r}_{13}$

Relation (2) also allows calculating $\boldsymbol{J}_{124}, \boldsymbol{J}_{134}, \boldsymbol{J}_{234}$ through each face of the tetrahedron. The Cartesian coordinates of the total average current density $\boldsymbol{J}_{\text {av }}$ are determined by projecting the current normal to three faces into the cartesian coordinates. 


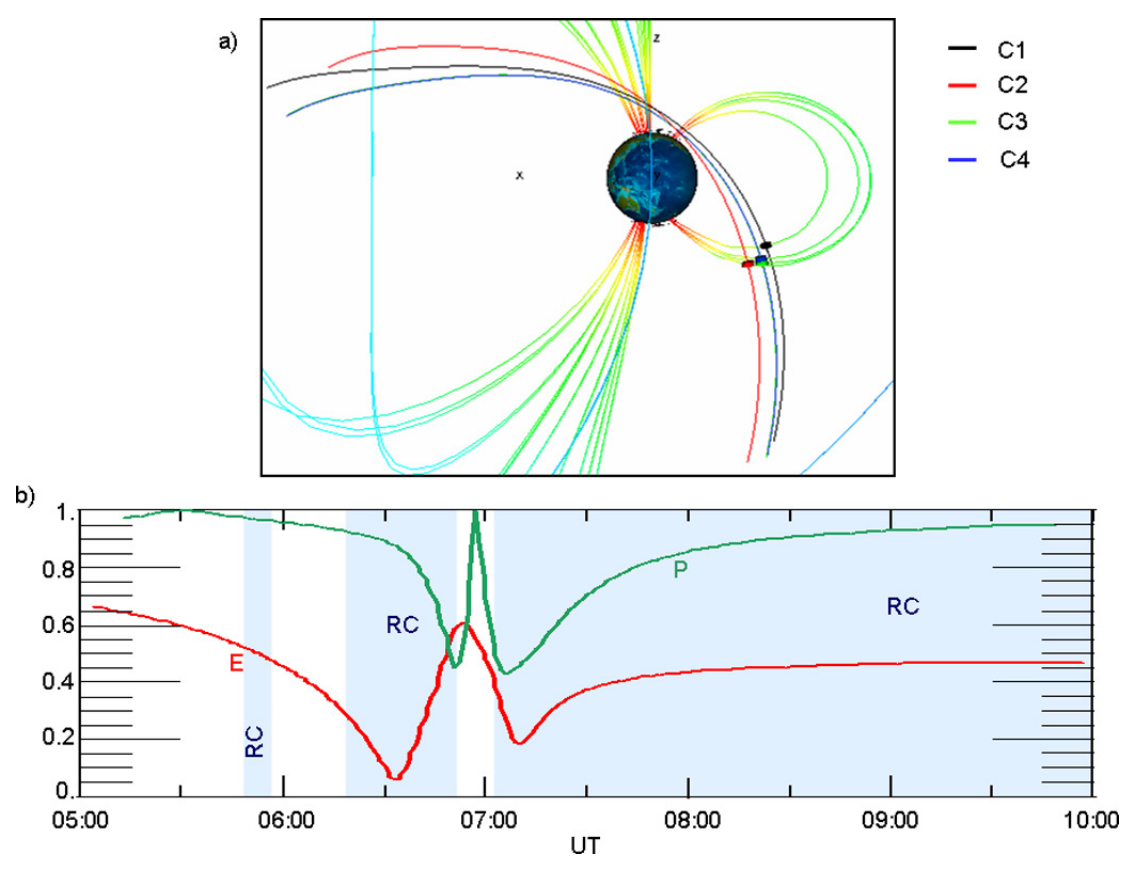

Fig. 2. (a) orbit of the four Cluster satellites for the 15 May 2009 ring current crossings. The colour code refers to the various satellites (black is C1, red is C2, green is C3 and blue is C4). The configuration of the tetrahedron at 06:00 UT can be seen in (a). (b) elongation $E$ (in red) and planarity $P$ (in green) of the tetrahedron. The blue shaded areas indicate the time intervals when the ring current is observed in $\mathrm{C} 1$ data.

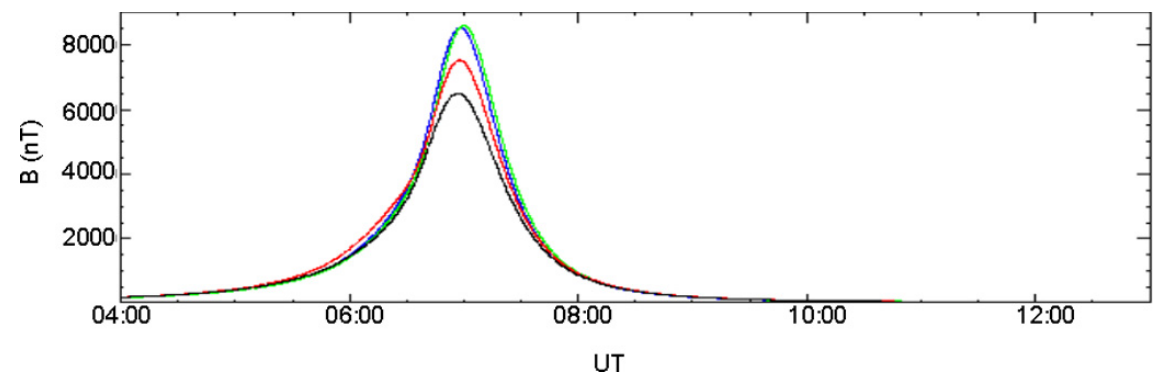

Fig. 3. Evolution of the magnetic field measured by the FGM instrument on board the four satellites. The colour code refers to the various satellites (black is $\mathrm{C} 1$, red is $\mathrm{C} 2$, green is $\mathrm{C} 3$ and blue is $\mathrm{C} 4$ ).

\subsection{Accuracy of the method}

The error sources of the curlometer have been studied by Dunlop et al. (2002), Chanteur (1998) and Robert et al. (1998a) and summarized by Vallat et al. (2005). They come from the assumptions made to obtain relation (2) and the usage of four separated satellites.

First, the two main assumptions of this technique are the linear variation of the magnetic field so that $\boldsymbol{J}_{\mathrm{av}}$ is constant over the tetrahedron and the stationarity over the studied current sheet. As the non-stationarity of the medium leads to the generation of nonlinear gradients in the magnetic field, the only point to check here is the linear variation of the magnetic field. To do so, Dunlop et al. (1988) showed that it is possible to calculate an estimate for $\operatorname{div} \boldsymbol{B}$ from: $\operatorname{div} \boldsymbol{B}\left|\Delta \boldsymbol{r}_{i k} . \Delta \boldsymbol{r}_{j k} \times \Delta \boldsymbol{r}_{j l}\right|=\left|\sum_{\text {cyclic }} \Delta \boldsymbol{B}_{i k} . \Delta \boldsymbol{r}_{j k} \times \Delta \boldsymbol{r}_{j l}\right|$

It is well known that $\boldsymbol{B}$ is solenoidal (Maxwell-Thomson relation: $\operatorname{div} \boldsymbol{B}=0$ ). Dunlop et al. (1988) showed that the calculation of $\operatorname{div} \boldsymbol{B}$ using relation (3) produces non-zero values that result from the nonlinear gradients, neglected in the estimation. As a consequence, the linear variation of the magnetic field can be checked by calculating $\operatorname{div} \boldsymbol{B}$, which, in the ideal case, should be zero. Nevertheless, we have to notice here that, if $\operatorname{div} \boldsymbol{B}$ is a nice indicator of the error on $\boldsymbol{J}_{\mathrm{av}}$, it is not proportional to it. Moreover, if any non-zero results indicate a bad estimation of $\boldsymbol{J}_{\mathrm{av}}$, a $\operatorname{div} \boldsymbol{B}$ close to zero is not an adequate condition for an accurate value of $\boldsymbol{J}_{\mathrm{av}}$. A better quality factor has been shown to be $\operatorname{div} \boldsymbol{B} / \operatorname{curl} \boldsymbol{B}$, as it 
is dimensionless and as it depends on the difference between the magnitude of $\operatorname{div} \boldsymbol{B}$ and $\boldsymbol{c u r l} \boldsymbol{B}$. It has been stated that $\operatorname{div} \boldsymbol{B} / \boldsymbol{c u r l} \boldsymbol{B} \ll 1$ indicates a good estimate of the average current density whereas $\operatorname{div} \boldsymbol{B} / \boldsymbol{c u r l} \boldsymbol{B} \geq 1$ indicates a standard deviation higher than $100 \%$.

Second, the magnetic field is measured by four satellites forming a tetrahedron, which can have many different shapes. It has been shown by Robert et al. (1998a) that only particular shapes can lead to the calculation of the $\boldsymbol{J}_{\mathrm{av}}$. The size of the tetrahedron has to be small enough so as to permit the gradients (inside the tetrahedron) to be as linear as possible. However, the smaller the tetrahedron is, the larger the absolute error made on $\Delta \boldsymbol{B}$ and $\Delta \boldsymbol{r}$ estimation is, and the more important the resulting error on $\boldsymbol{J}_{\mathrm{av}}$ is too. Moreover, a too elongated or a too planar tetrahedron will lead to important errors on the estimation of some of the $\boldsymbol{J}_{\mathrm{av}}$ components and will decrease the accuracy of the method.

\section{Event presentation}

\subsection{Cluster orbit and instrumentation}

The Cluster mission is based on four identical spacecraft (denoted C1, C2, C3 and C4) launched on similar elliptical polar orbits. Figure $2 \mathrm{a}$ presents the orbit of the four Cluster satellites for the 15 May 2009 ring current crossings. The colour code refers to the various satellites (black is $\mathrm{C} 1$, red is $\mathrm{C} 2$, green is $\mathrm{C} 3$ and blue is $\mathrm{C} 4)$. The magnetic equator is crossed at 06:29 UT (C1), 06:33 UT (C2 and C4) and 06:35 UT (C3). At the beginning of the mission, the perigee was at $4 R_{\mathrm{E}}$ and the apogee at 19.6 $R_{\mathrm{E}}$ (Escoubet et al., 2001). Due to orbital perturbations and drag from the exosphere, the perigee decreases slowly and it was about $2 R_{\mathrm{E}}$ during the time interval under study. The configuration of the tetrahedron at 06:00 UT can be seen in Fig. 2a. The four Cluster satellites had a separation of $1000 \mathrm{~km}$ when crossing the auroral zone area. $\mathrm{C} 1$ was leading and then came $\mathrm{C} 2, \mathrm{C} 4$ and $\mathrm{C} 3$ brings up the rear. The shape of the tetrahedron evolves along the trajectory of the spacecraft. To characterise the geometrical shape of the tetrahedron during the time interval under study, Fig. 2b presents the elongation $E$ (in red) and the planarity $P$ (in green) of the tetrahedron between 05:00 UT and 10:00 UT (see Robert et al., 1998b, for a definition of these two factors). $E$ and $P$ lie between 0 and 1 . As shown by Robert et al. (1998b), $E=1$ means the four satellites are located along a straight line whatever $P$ is (1-D tetrahedron), $P=1$ means the four satellites are located in a plane whatever $E$ is (2-D tetrahedron), and $E=0$ means the satellites are located in the circumference of a circle if $P=1$ (2-D tetrahedron) and of a sphere if $P=0$. As shown in Fig. 2 b, $E$ varies between 0.05 and 0.6 during the whole observation when $P$ lies between 0.4 and 1 . As a consequence, the satellites are located in the surface of a more or less elongated ellipsoid, and in a plane at 05:30 UT and 06:57 UT.
Eleven experiments on board each spacecraft allow a wide variety of measurements of the plasma parameters (particles and fields). Among them, a fluxgate magnetometer (FGM), as well as two ion spectrometers (HIA and CODIF), are present. We will now present the data of these two instruments for the ring current crossings under study as well as the geomagnetic activity level.

\subsection{FGM data and geomagnetic activity}

The FGM experiment on board Cluster consists of two triaxial fluxgate magnetometers and an onboard data-processing unit on each spacecraft (Balogh et al., 1997, 2001). High vector sample rates (up to 67 vectors $\mathrm{s}^{-1}$ ) at high resolution (up to $8 \mathrm{pT}$ ) allow for a precise measurement of the ambient magnetic field. The background interference from the spacecraft is minimised by the positioning of the magnetometers on a five metre boom, which avoids interference from the spacecraft. The magnetic field component along the spin axis (which is almost perpendicular to the ecliptic) will carry the main part of the error made on the measurement, because offsets in the spin plane measurements are easily removed by noting spin-period oscillations. In addition to the on-ground calibrations made to determine the expected maximal offset on each spacecraft (up to $0.1 \mathrm{nT}$ ), in-flight calibrations of the magnetometers are regularly applied in order to maximise the accuracy of the magnetic field measurements.

Figure 3 presents the evolution of the magnetic field measured by the FGM instrument on board the four satellites between 04:00 UT and 13:00 UT. As shown in Fig. 2a, the satellites cross the inner magnetosphere from the Southern Hemisphere to the northern one. During the orbit, the magnetic field measured on board each satellite increases reaching a maximum in the cusp region (Fig. 3). Afterwards, the magnetic field decrease when the satellites travel toward the night side.

Figure 4 presents the AU (top panel), AL (middle panel) and Dst (bottom panel) indices evolution between the 14 May 2009 at 08:00 UT and the 16 May 2009 at 08:00 UT. The orange dashed lines are located 04:00 UT and at 13:00 UT and delimitate the time range of the magnetic field data presented in Fig. 3. A variation of the auroral indices is observed before the observation (blue arrows). As a reminder, the AU index measures the intensity of the eastward electrojet which is part of the region 2 current system, while the AL index measures the intensity of the westward electrojet which is part of the region 1 current system. The region 2 current system closes in the night side, close to the magnetic equator at $L \approx 4 R_{\mathrm{E}}$. The region 1 current system closes in the dayside magnetopause. The increase of these two indices indicates a compression of the day side magnetopause and an injection of particles in the night side. The auroral indices evolution is associated with a very smooth variation of the Dst index which reaches a very high minimum at $-18 \mathrm{nT}$. These observations show that the ring current is 


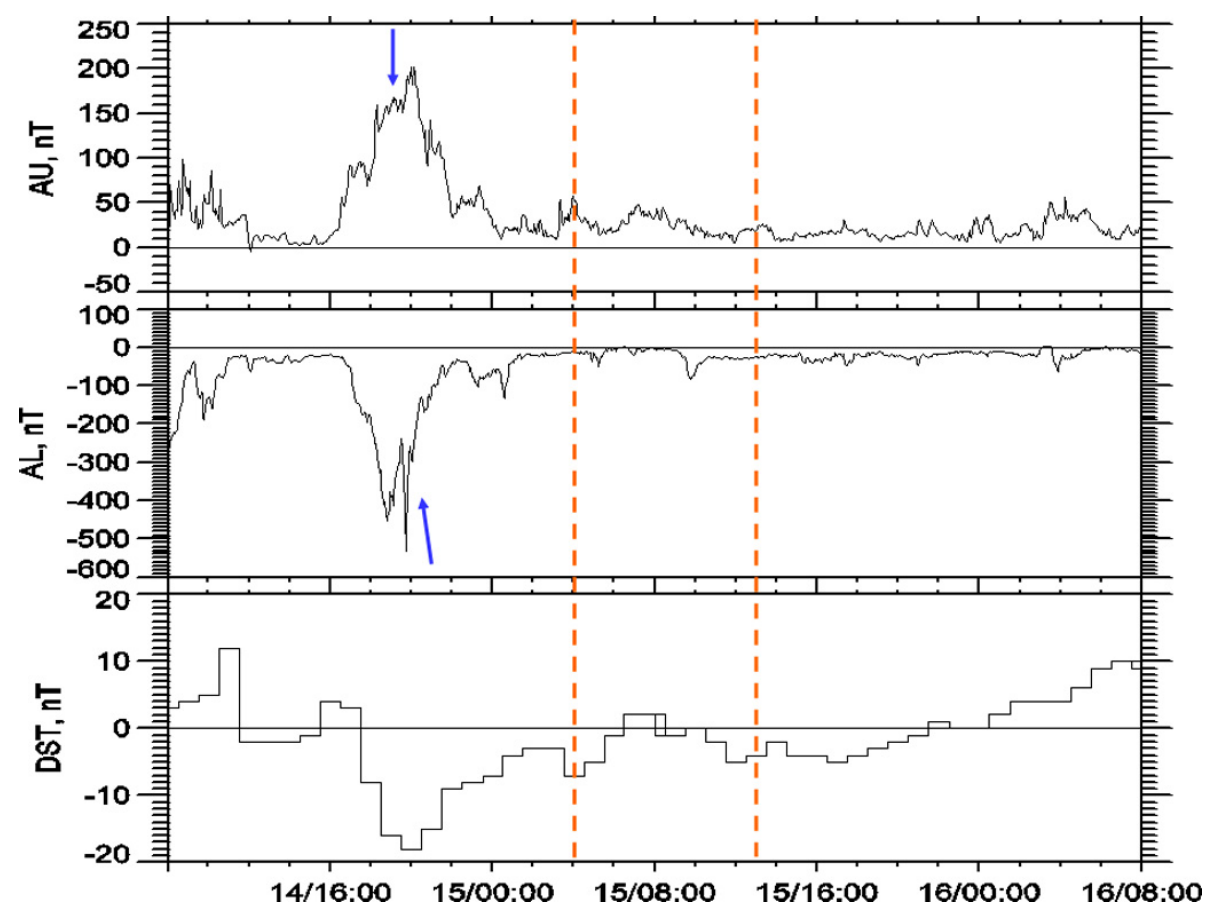

Fig. 4. AE (top panel), AU (middle panel) and Dst (bottom panel) indices evolution between the 14 May at 08:00 UT and the 16 May 2009 at 08:00 UT. The orange dashed lines are located on 15 May at 04:00 UT and at 13:00 UT and delimitate the time range of the magnetic field data presented in Fig. 3. The blue arrows indicate variations of the auroral indices.

only very slightly disturbed by the injection of particles and the magnetic field compression. To summarize, variations in the solar wind are responsible for the injection of particles and the compression of the magnetosphere, but they are weak enough to cause no more than a very low disturbance of the ring current.

\subsection{CIS data}

The Cluster Ion Spectrometer experiment consists of two complementary ion sensors, the COmposition and Distribution Function analyzer (CODIF) and the Hot Ion Analyzer (HIA). CODIF gives a mass per charge composition with a $22.5^{\circ}$ angular resolution and HIA offers a better angular resolution $\left(\sim 5^{\circ}\right)$, but without mass discrimination. CIS is capable of measuring the full three-dimensional ion distribution of the major ion species, from thermal energies $\left(\sim 1 \mathrm{eV} \mathrm{q}^{-1}\right)$ to about $40 \mathrm{keV} \mathrm{q}^{-1}$, with one spacecraft spin (4 s) time resolution (Rème et al., 2001).

Figure 5 presents data from the HIA instrument on board $\mathrm{C} 1$ as energy-time spectrograms. As the spacecraft observe similar states of the ring current, the spectrograms are very similar from a satellite to another (not shown), and they are just shifted in time according to the orbital shift of the spacecraft. Figure 5a shows the ion data from 05:30 UT to 10:00 UT and Fig. 5b presents a zoom between 05:30 UT and 07:15 UT. The different populations observed in the spectrograms allow determining which part of the magnetosphere is crossed by the satellite. At the beginning $\mathrm{C} 1$ leaves the southern cusp region to enter into the inner magnetosphere (see Fig. 5a). It crosses a transition zone indicated by particle injections characteristic of the near-Earth plasma sheet (TZ in Fig. 5b). Between 05:32 UT and 05:38 UT it crosses the Alfvén boundary layer to enter in the part of the magnetosphere where plasma corotation dominates (Alfvén, 1968; Volland, 1973; Stern, 1975). The satellite also enters into the inner magnetosphere, where the corotation electric field is higher than the convection one. The ring current (annotated RC in Fig. 5) and the sub keV population (annotated SP in Fig. 5) are observed, respectively, above and below $10 \mathrm{keV}$ between 05:38 UT and 05:55 UT. The ring current appears structured in two energy bands and it is the part of the ring current ion population covered by the CIS energy range. These are the signature of an ion spectral gap (McIlwain, 1972; Sauvaud et al., 1998a, b) which has been shown to be the result of particle drift under the global large scale electric field (Vallat et al., 2007; McIlwain, 1972; Buzulukova, 2002). They represent the signature of a quiet ring current, which is in agreement with the observations on magnetic indices presented in Sect. 3.2. Between 05:44 UT and 05:56 UT, 06:15 UT and 06:48 UT and 06:55 UT and 07:05 UT, a background can be observed in the spectrogram (annotated OB and IB in Fig. 5b). It comes from the outer $(\mathrm{OB})$ and the inner (IB) radiation belt particles which can go through the instrument walls and directly hit the detectors. 


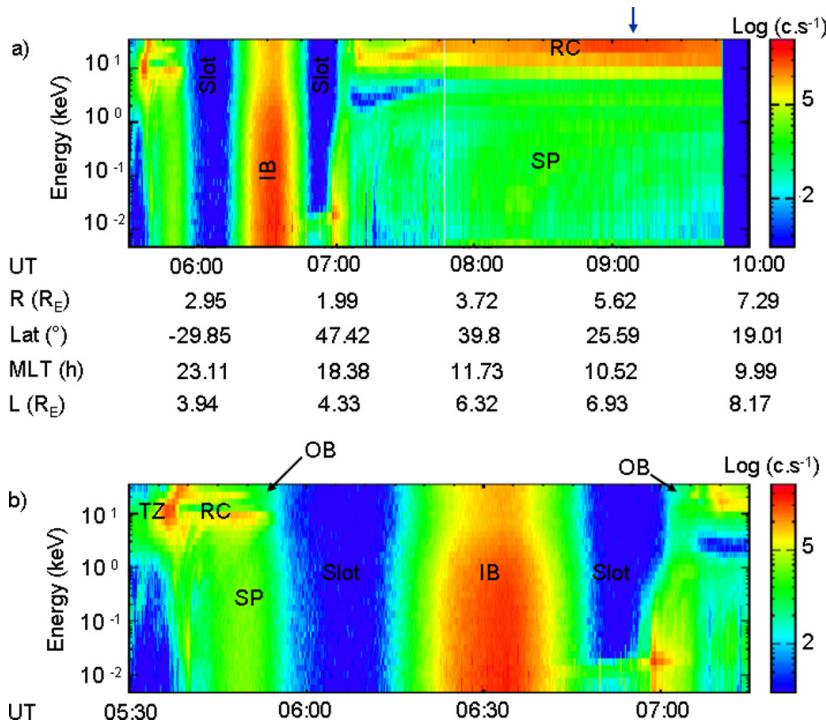

Fig. 5. Data from the CIS/HIA instrument on board $\mathrm{C} 1$ for the 15 May 2009 perigee pass as energy-time spectrograms, in ion counts (corrected for the detection efficiency) per second. (a) during the whole time interval under study, (b) during the perigee pass. In each plot, TZ: transition zone, RC: ring current, SP: sub-keV population, IB and $\mathrm{OB}$ : inner and outer radiation belts.

This background appears as an increase of the count rate at all energies in the ion spectrogram and can be used to study the radiation belts boundaries (Ganushkina et al., 2011). On each side of the inner belt the satellite crosses the slot region characterised by very low count rates in the spectrograms (Fig. 5a and b). During the slot crossing, the satellite crosses the terminator and encounters again the ring current and the sub $\mathrm{keV}$ population (RC and SP in Fig. 5a) at 07:00 UT. To summarize, the ring current ion population is observed twice during the event under study: first between 05:38 UT and 05:55 UT, in the dawn side, at about $3.5 R_{\mathrm{E}}$ and in a $\left(-40.7^{\circ}\right.$, $-33.1^{\circ}$ ) magnetic latitude range, from 07:00 UT, in the dusk side, in a $\left(2 R_{\mathrm{E}}, 7.5 R_{\mathrm{E}}\right)$ geocentric distance range and in a $\left(18.5^{\circ} ; 47.4^{\circ}\right)$ magnetic latitude range. The radiation belts are the higher energy population of the ring current. As a consequence, we define here the ring current region as the part of the orbit where the satellite encounters the ring current and the radiation belts population. It enters inside this region at 05:38 UT and doesn't leave it in the time interval under study. The homogeneous blue area observed from 09:48 UT indicates that the instrument has been switched off and that there is no data in this part of the orbit. We note that the higher-energy part of the ring current (above $\sim 40 \mathrm{keV} \mathrm{q}^{-1}$ ) is not detected by CIS.

\subsection{Estimation of the current density along the Cluster orbit}

We will now focus on the calculation of the current density using the curlometer technique. First, this method is valid only for a steady medium. On 15 May 2009, the ring current is a steady one all along the observation. Second, the four satellites have to be located in the same current sheet. $\mathrm{C} 1$ is leading. It is located inside the ring current region from 05:38 UT. C 3 brings up the rear and is located in the ring current region from 05:48 UT. Therefore, the four satellites are in the same current sheet from 05:48 UT.

Figure 6 presents the results from the curlometer. The blue shaded areas indicate the time intervals when the ring current population is observed in $\mathrm{C} 1$ data. These blue areas can also be seen on Fig. 2b. The L-shell and the magnetic latitude of $\mathrm{C} 1$ is given in Fig. 6. As the current density is calculated using the four satellites, these values are indicative and only give an average position. Frame a shows the time evolution of $\left|\boldsymbol{J}_{\mathrm{av}}\right|$ and its components in cylindrical coordinates. We can notice first that $\boldsymbol{J}_{\mathrm{av}}$ and its components go to infinity before entering inside the ring current (between 05:25 UT and 05:36 UT) and during the second slot region crossing (at about 06:55 UT). This strong increase is linked with a strong increase of $\operatorname{div} \boldsymbol{B}$ (frame b, top panel) which also goes to infinity at the same time intervals. This observation indicates a very bad accuracy of $\boldsymbol{J}_{\mathrm{av}}$ estimation in this part of the orbit. Moreover, $P=1$ at 05:30 UT and at 06:57 UT (see Fig. 2). As a consequence, the four satellites are located in the same plane and the errors made on the estimation of some of the $\boldsymbol{J}_{\text {av }}$ components are quite important. Some oscillations (violet arrows) are observed between 05:30 UT and 05:48 UT on $\boldsymbol{J}_{\text {av }}$ and on its components. These oscillations are also observed on $\operatorname{div} \boldsymbol{B}$. A more detailed study of the magnetic field data allows showing that they come from Pc5 ULF pulsations, which are responsible for oscillations in the magnetic field lines. A more detail study of this phenomenon is out of the scope of the paper.

From 05:48 UT the four satellites are located in the ring current region. $\boldsymbol{J}_{\text {av }}$ calculated using the curlometer is equal or higher than $100 \mathrm{nA} \mathrm{m}^{-2}$ between the two strong increasing of $\boldsymbol{J}_{\mathrm{av}}$ and close to $200 \mathrm{nA} \mathrm{m}^{-2}$ after the second strong increasing of $\boldsymbol{J}_{\mathrm{av}}$. Focusing now on the $\boldsymbol{J}_{\mathrm{av}}$ components, azimuthal, radial and parallel to the $\mathrm{z}$-axis currents are observed all along the ring current region crossing. In the cylindrical coordinates, the ring current flows in the azimuthal direction. It flows eastward if $\boldsymbol{J}_{\mathrm{av} \varphi}$ is positive and westward if $\boldsymbol{J}_{\mathrm{av} \varphi}$ is negative. The current in the radial and parallel to the $\mathrm{z}$-axis directions indicate the existence of radial and/or field aligned currents. The average current density in the azimuthal direction obtained using the curlometer is negative until 06:03 UT, indicating a westward ring current. At 06:03 UT, the azimuthal current direction changes to become eastward at $L=3.59 R_{\mathrm{E}}$. This result is in agreement with the past results which locate the current reversal boundary 


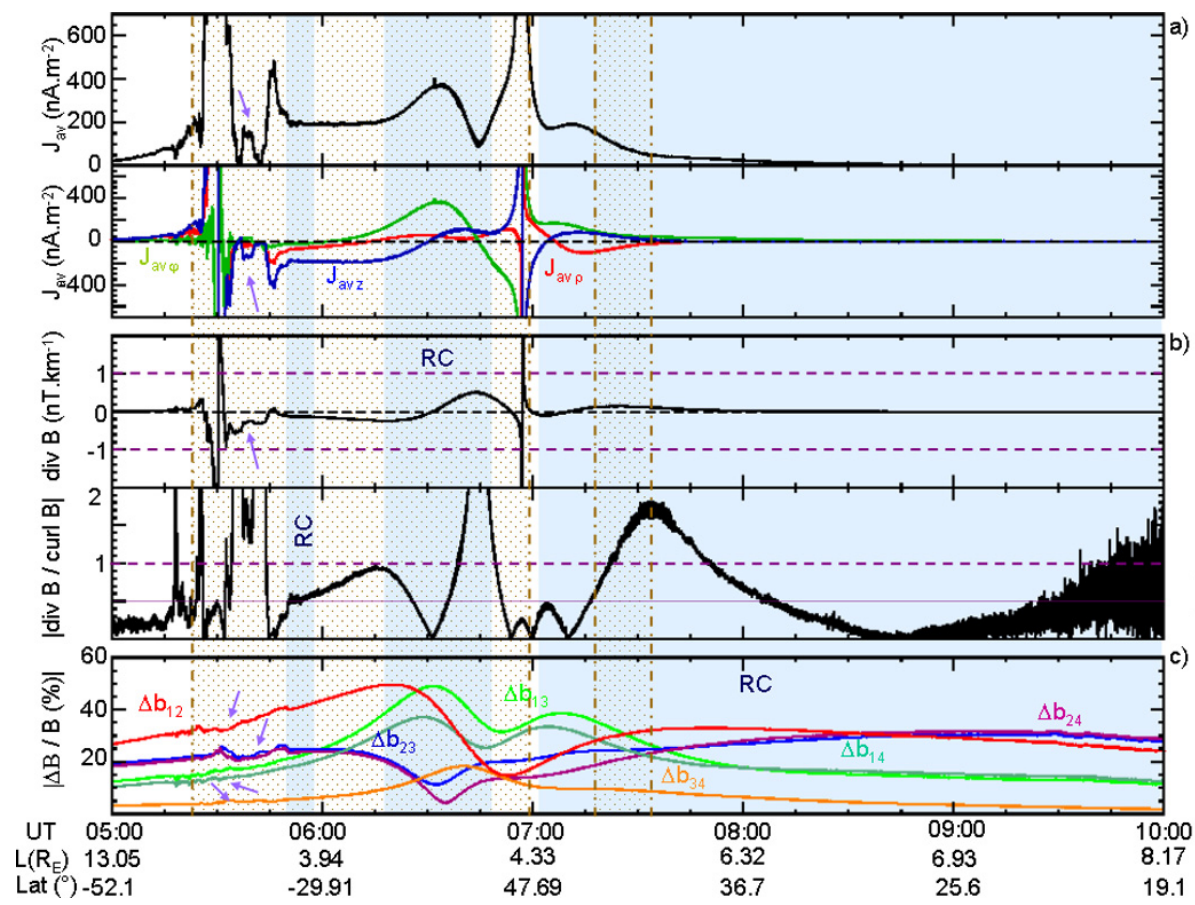

Fig. 6. Estimation of the current density along the Cluster orbit on 15 May 2009 ring current region crossing. Frame (a): time evolution of $\left|\boldsymbol{J}_{\mathrm{av}}\right|$ (top panel) and its components in cylindrical coordinates (bottom panel). Frame (b): $\operatorname{div} \boldsymbol{B}$ and $|\operatorname{div} \boldsymbol{B} / \boldsymbol{c u r l} \boldsymbol{B}|$. Frame $(\mathbf{c}): \Delta b_{i j}=$ $B_{i}-B_{j} / B$, with $i$ and $j$ the satellite number (panel a). In each frame, the blue shaded areas indicate the time intervals when the ring current is observed on $\mathrm{C} 1$ data. The brown dashed lines are located where $|\operatorname{div} B|=1 \mathrm{nT} \mathrm{km}^{-1}$. The L-shell and the magnetic latitude of $\mathrm{C} 1$ is given in Fig. 6. As the current density is calculated using the four satellites, these values are indicative and only give an average position.

between $3 R_{\mathrm{E}}$ and $4 R_{\mathrm{E}}$ (Le et al., 2004; Jorgensen et al., 2004). Then the average current density increases to reach $360 \mathrm{nA} \mathrm{m}^{-2}$ at the perigee pass. After the second slot region crossing, the average current density in the azimuthal direction is eastward and about equal to $170 \mathrm{nA} \mathrm{m}^{-2}$. Then, it decreases to reach zero at 09:17 UT $\left(L=7.3 R_{\mathrm{E}}\right)$. It never becomes westward.

What about the accuracy of this result? In Fig. 6, the brown dotted-dashed lines indicates the time when $|\operatorname{div} B|=$ $0.1 \mathrm{nT} \mathrm{km}^{-1}$. Between two lines, in the dotted regions, $|\operatorname{div} \boldsymbol{B}|>0.1 \mathrm{nT} \mathrm{km}^{-1}$, so $\operatorname{div} \boldsymbol{B}$ is much greater than zero. As a consequence, the Maxwell-Thomson law $(\operatorname{div} \boldsymbol{B}=0)$ is violated during these parts of the orbit and the average current density obtained from the curlometer may be inaccurate. In the other part of the orbit, $\operatorname{div} \boldsymbol{B}$ is close to zero. As shown during the development of the method, a $\operatorname{div} \boldsymbol{B}$ close to zero is a necessary but not adequate condition for an accurate value of $\boldsymbol{J}_{\mathrm{av}}$. A better quality factor has been shown to be $\operatorname{div} \boldsymbol{B} /$ curl $\boldsymbol{B}$ as it is dimensionless. Moreover, the magnetic field has to change slowly from a satellite to another. $|\operatorname{Div} B / \operatorname{curl} B|$ is presented in the second panels of frame $\mathrm{b}$ in Fig. 6. It is noticeable that $|\operatorname{Div} B / \operatorname{curl} B|$ is very noisy during part of the orbit, which may be due to the error on the magnetic field measurement. Frame c (Fig. 6) presents the time evolution of $\Delta b_{i j}$ (where $\Delta b_{i j}=\left(B_{\mathrm{C} i}-B_{\mathrm{C} j}\right) / B$, with $i$ and $j$ the satellite number). We can notice first that the same oscillations as the ones observed on $\operatorname{div} \boldsymbol{B}$, on $\boldsymbol{J}_{\text {av }}$ and on its components are seen on $\Delta b_{i j}$, which confirm that they come from oscillations on the magnetic field lines. During the main part of the ring current region crossing, $\operatorname{div} \boldsymbol{B} /$ curl $\boldsymbol{B}$ is above 0.5 (dotted purple line), indicating a standard deviation higher than $50 \%$, and there is always a $\Delta b_{i j}$ higher than $25 \%$. As a consequence, the curlometer technique is not valid during the whole ring current crossing, even when $|\operatorname{div} \boldsymbol{B}|<0.1 \mathrm{nT} \mathrm{km}^{-1}$.

\section{Study of the accuracy of the curlometer technique along the Cluster orbit}

\subsection{Current density from the Tsyganenko model}

In this paper, the curlometer technique has been used to calculate the current density between 2 and $7 R_{\mathrm{E}}$. It has been supposed that the ring current is stable and that the four satellites observe the same state of the ring current. This hypothesis allows calculating the current density and the divergence of the magnetic field. The results show that the curlometer technique is not valid for the whole ring current region crossing. To calculate the current density, the magnetic field data and the position of the satellites have been used. The effects 


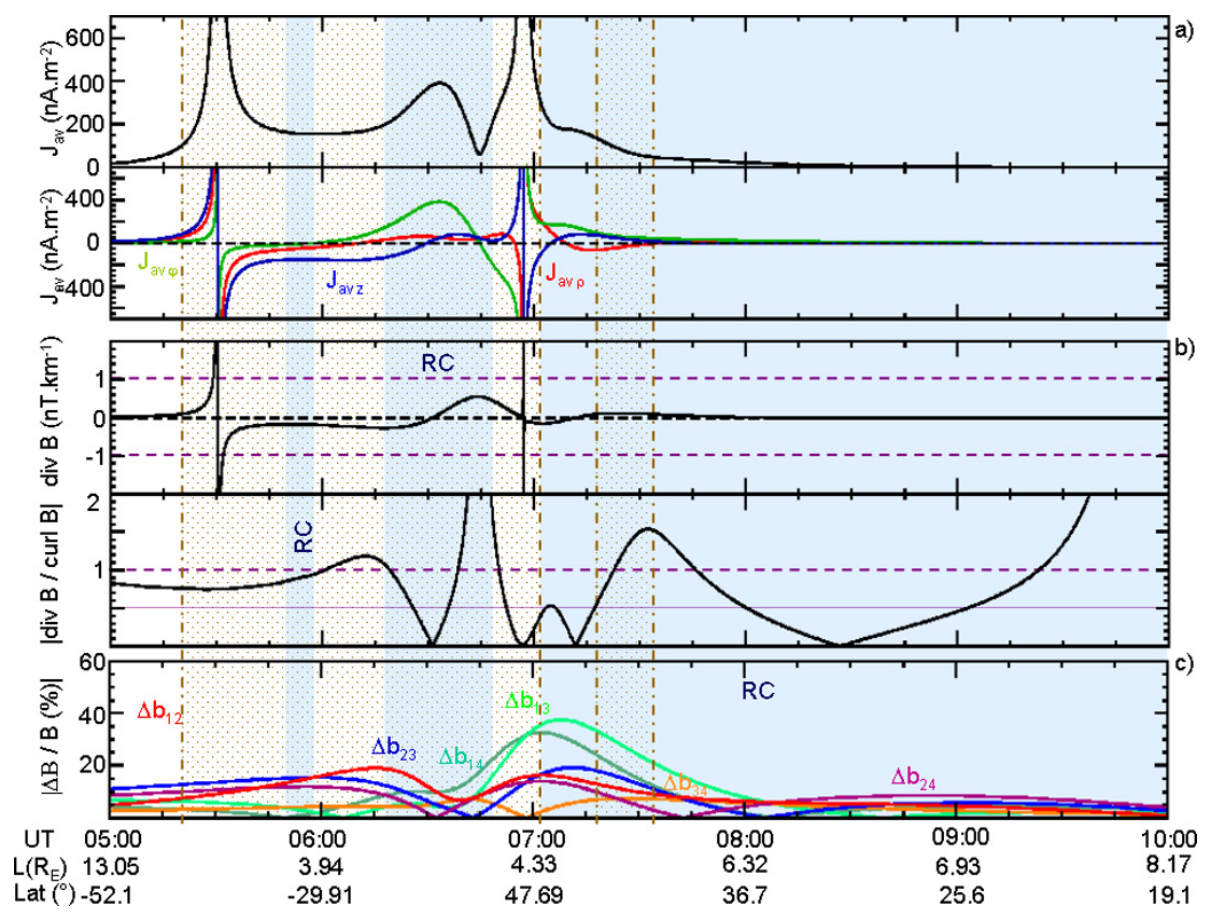

Fig. 7. Same as Fig. 6, but using a Tsyganenko model.

of the measurement errors have not been mentioned in the previous part, but it has been shown that these errors increase strongly the uncertainty of $\boldsymbol{J}_{\mathrm{av}}$ (Dunlop et al., 2002; Vallat et al., 2005). Moreover, it has been shown that variations in the solar wind are responsible for injections of particles and for compression of the magnetosphere. The Dst index variations associated with increasing auroral indices is very smooth. As stated above, this observation assumes that the ring current is not (or is only very slightly) disturbed. However, changes in the ring current are responsible for distortion of the magnetic field lines and will impact the results obtained using the curlometer technique. In order to suppress those uncertainties, a Tsyganenko model (Tsyganenko and Stern, 1996) has been used to calculate the magnetic field along the Cluster orbit and to test the curlometer technique along this orbit. Using a model, the position of the satellites and the magnetic field is known without errors. Moreover, the parameters of the model have been defined to model a quiet magnetosphere with no injection of particles and no compression of the magnetopause. As a consequence, the satellites cross a non-disturbed ring current. The results obtained using the curlometer technique in such a magnetic field for the 15 May 2009 perigee pass is presented in Fig. 7, which is similar to Fig. 6. The $\Delta b_{i j}$ obtained using the Tsyganenko model show some differences from the ones obtained using the FGM magnetic field, but the evolutions and the magnitudes are similar. Nevertheless, we have to notice first that they are slightly smaller as the maximum of the $\Delta b_{i j}$ for the FGM data is $50 \%$ when it is $37 \%$ for the Tsyganenko model, and second that the oscillations observed before the entrance in the ring current region in the FGM data are not observed in the Tsyganenko magnetic field, as they come from the variation of the magnetospheric activity. Looking now at $\boldsymbol{J}_{\mathrm{av}}$ and $\operatorname{div} \boldsymbol{B}$, the temporal evolution of $\boldsymbol{J}_{\mathrm{av}}$ and $\operatorname{div} \boldsymbol{B}$ is similar to the ones obtained using the FGM data. In particular, they go to infinity at the same time because of the planar tetrahedron, currents in the azimuthal, radial and parallel to the z-axis current are observed, and the azimuthal current is first westward and then eastward. As the one obtained using the FGM data the azimuthal current is eastward after the second slot region crossing and never becomes westward. A difference can be observed in the values of the current and of the $\operatorname{div} \boldsymbol{B}$, which are slightly smaller due to smaller $\Delta b_{i j}$.

What about the accuracy of this result? As in Fig. 6, the brown dotted-dashed lines in Fig. 7 indicates the time when $|\operatorname{div} B|=0.1 \mathrm{nT} \mathrm{km}^{-1}$ and the dotted regions, the time when the Maxwell-Thomson law $(\operatorname{div} \boldsymbol{B}=0)$ is violated. The dotted part obtained for the model and the data are about the same. Comparing now the $\operatorname{div} \boldsymbol{B} / \operatorname{curl} \boldsymbol{B}$, it is noticeable that the one obtain using the Tsyganenko model is less noisy than the one obtained using the FGM data. This observation highlights an interesting point: it shows that this noise comes from the errors on the magnetic field data and on the satellite position. Nevertheless, the evolution and the magnitudes obtained are quite similar for the two magnetic fields. Finally, there is always a $\Delta b_{i j}$ higher than $10 \%$, which is smaller than the limit obtained for the FGM data. Therefore, the accuracy looks to be better for the Tsyganenko model, but the 


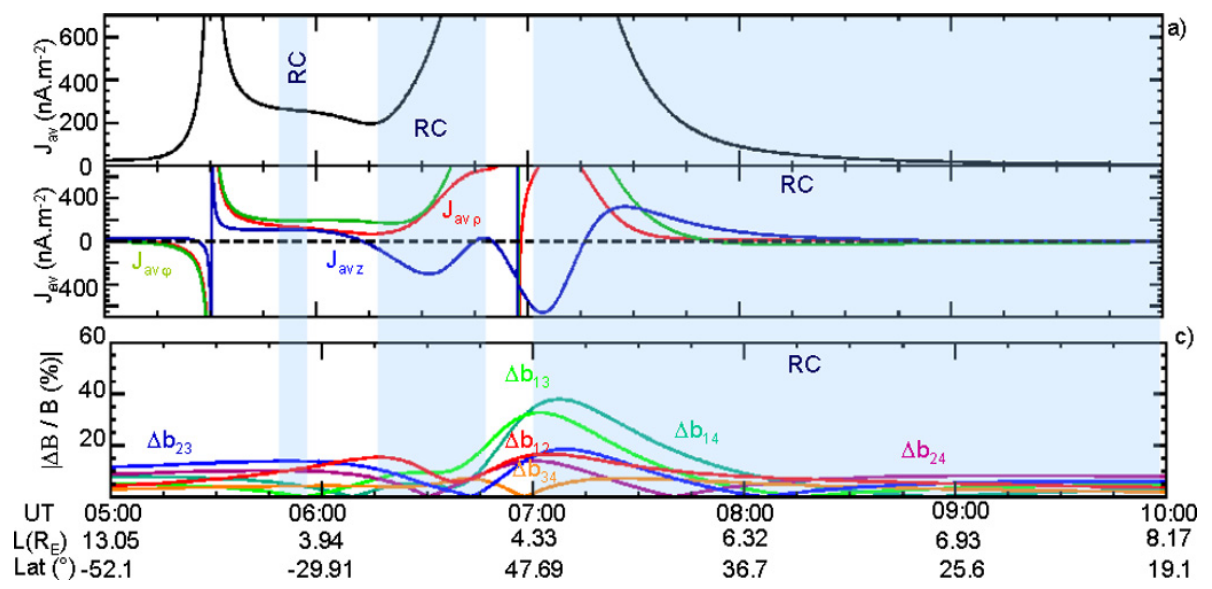

Fig. 8. Estimation of the current density and $\Delta b_{i j}$ along the Cluster orbit on 15 May 2009 ring current region crossing for a dipolar model. In each frame, the blue shaded areas indicate the time intervals when the ring current is observed on $\mathrm{C} 1$ data.

uncertainties on the results are still too important and the curlometer technique is not valid during the whole ring current region crossing.

\subsection{Current density from the dipolar model}

In Sect. 4.1, the curlometer technique has been used on a Tsyganenko model. The results obtained show that the low accuracy of the method on the 15 May 2009 doesn't come from nonlinear gradients induced by the magnetospheric activity, nor from the uncertainties on the position of the satellites or on the magnetic field data. In the Tsyganenko model, the magnetic field is made up of an internal and an external magnetic field. The internal magnetic field chosen in this study is a dipolar one. The external magnetic field comes from the magnetic field induced by the magnetospheric currents as the magnetopause current or the ring current. By definition, the current calculated using the Maxwell-Ampere's law for a dipolar magnetic field has to be equal to zero. Non-zero values would be representative of errors induced by the curlometer technique. Using a Cluster orbit, Vallat et al. (2005) show that this error doesn't affect significantly the current density calculation. To do so, they used two current densities obtained at a perigee located at $4 R_{\mathrm{E}}$. On 15 May 2009, the perigee was located at about $2 R_{\mathrm{E}}$ and it is plausible that the effect of the dipolar model on the $\boldsymbol{J}_{\text {av }}$ calculation is more important. In order to address this question, the curlometer has been used to calculate $\boldsymbol{J}_{\text {av }}$ using a dipolar model. The obtained $\boldsymbol{J}_{\mathrm{av}}$ and its components obtained are presented in Fig. 8 (frame a) as well as $\Delta b_{i j}$ (frame b). As in Figs. 2, 6 and 7 the blue shaded areas indicate the time intervals when the ring current population is observed in $\mathrm{C} 1$ data. A strong current is observed during the main part of the crossing. This current is in the azimuthal, radial and parallel to the $\mathrm{z}$-axis direction. This result indicates a strong contribution of the dipole to the current calculation for this event, which is, therefore, not reliable. Moreover, the $\Delta b_{i j}$ obtain using the dipolar model is very similar to the ones obtain using the Tsyganenko model and no clear differences are visible. As a consequence, the very bad accuracy of the method for this event may come from nonlinear gradients induced by the distance between the satellite and the quick evolution of the magnetic field close to the Earth.

\subsection{Current density from the distortion of the Tsyganenko magnetic field}

It has been shown in the previous section that the dipolar magnetic field induces a strong current in the $\boldsymbol{J}_{\text {av }}$ calculation. As a consequence, $\boldsymbol{J}_{\mathrm{av}}=\boldsymbol{J}_{\mathrm{av} \mathrm{dip}}+\boldsymbol{J}_{\mathrm{av} \text { RC}}$, where $\boldsymbol{J}_{\text {av dip }}$ is the current density induced by the dipole, and $\boldsymbol{J}_{\mathrm{av}} \mathrm{RC}$ is the current density from the ring current itself. In order to reduce the error induced on the curlometer results, we will now calculate the current density for a magnetic field obtained by subtracting the dipolar magnetic field from the Tsyganenko one. The results obtained using the curlometer technique in such a magnetic field for 15 May 2009 perigee pass are presented in Fig. 9, which is similar to Fig. 6. The $\Delta b_{i j}$ obtain using the new model show some differences from the ones obtained using the others magnetic field. The $\Delta b_{i j}$ is below $8 \%$ except between 06:05 UT and 06:15 UT and is never above $30 \%$. As a consequence this magnetic field changes much more slowly than the dipolar, Tsyganenko and FGM magnetic fields. Looking now at $\boldsymbol{J}_{\mathrm{av}}$ and $\operatorname{div} \boldsymbol{B}$, their temporal evolution is very different from the evolutions observed for the FGM and the Tsyganenko magnetic fields, which confirm the importance of the error induced by the dipole in the current calculation. As for the FGM and the Tsyganenko magnetic fields, an azimuthal, a radial and a parallel to the zaxis current are observed. The azimuthal current is first westward. The current density in this direction is about constant and equal to $100 \mathrm{nA} \mathrm{m}^{-2}$ until 06:05 UT $\left(L=3.5 R_{\mathrm{E}}\right)$. Then, 


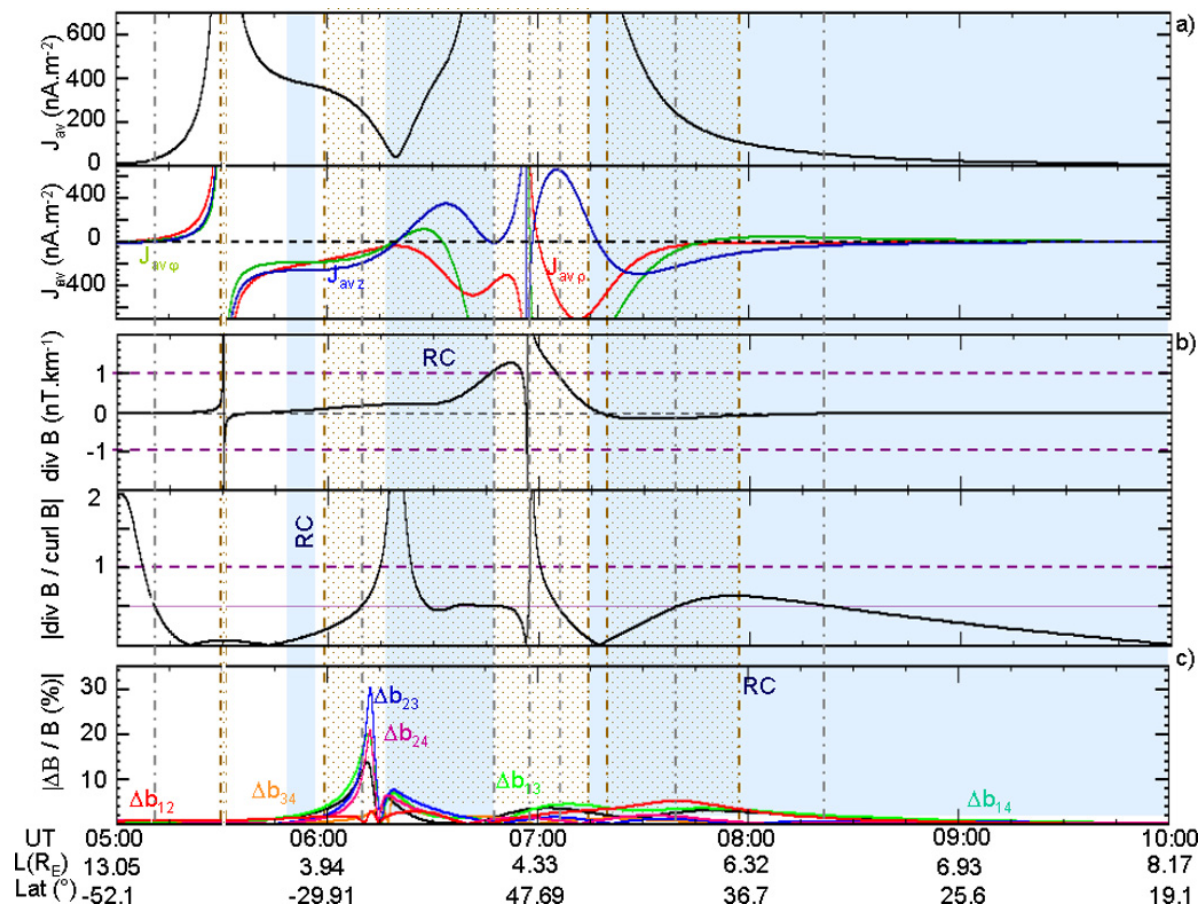

Fig. 9. Same as Fig. 6, but using a magnetic field calculated by subtracting a dipolar magnetic field from the Tsyganenko one. The grey dotted-dashed lines indicate the times when $\operatorname{div} B / \operatorname{curl} \boldsymbol{B}=5$.

it increases to turn eastward at 06:18 UT $\left(L=2.55 R_{\mathrm{E}}\right)$. As the one obtained using the FGM and the Tsyganenko magnetic fields, it is eastward after the second slot region crossing and never becomes westward again.

What about the accuracy of this result? As in Fig. 6, the brown dotted-dashed lines in Fig. 9 indicates the time when $|\operatorname{div} B|=0.1 \mathrm{nT} \mathrm{km}^{-1}$ and the dotted regions, the time when the Maxwell-Thomson law $(\operatorname{div} \boldsymbol{B}=0)$ is violated. The dotted part obtained for the model and the data are about the same. Looking now on the $\operatorname{div} \boldsymbol{B} / \operatorname{curl} \boldsymbol{B}$ temporal evolution, it is interesting to note that it is below 5 between 05:15 UT and 06:10 UT, 06:47 UT and 06:57 UT, 07:05 UT and 07:40 UT, and from 08:20 UT (grey dotted-dashed lines in Fig. 9 indicate those times). During each interval, it goes very close to zero, indicating a good accuracy of the method. Nevertheless, during part of the first and the third interval and during the whole second interval div $\boldsymbol{B}>0.1 \mathrm{nT} \mathrm{m}^{-2}$. Moreover, $\boldsymbol{J}_{\text {av }}$ increases strongly during the second and third interval indicating strong errors in the curlometer results. If we consider that $\operatorname{div} \boldsymbol{B}<0.1 \mathrm{nT} \mathrm{m}^{-2}$ indicates a good accuracy of the method, then, $\boldsymbol{J}_{\mathrm{av}}$ may be correct between 05:15 UT $\left(L=10.26 R_{\mathrm{E}}\right)$ and 06:10 UT $\left(L=3.07 R_{\mathrm{E}}\right)$ and from 08:20 UT ( $L=6.3 R_{\mathrm{E}}$ ). We will now study in more details the value, the current density and the orientation of the current for each time interval.

Looking first at $\boldsymbol{J}_{\text {av }}$ during the first interval, it goes to infinity until 05:30 UT and then decreases slowly from $400 \mathrm{nA} \mathrm{m}^{-2}$ to $350 \mathrm{nA} \mathrm{m}^{-2}$. Vallat et al. (2005) deter- mined for the 18 March 2002 perigee pass between $L=$ $4.2 R_{\mathrm{E}}$ and $5 R_{\mathrm{E}}$ and found $20 \mathrm{nAm}^{-2}<J_{\mathrm{av}}<30 \mathrm{nA} \mathrm{m}^{-2}$. On 15 May 2009, the satellites are located in a [3.07 $R_{\mathrm{E}}$; $7.77 R_{\mathrm{E}}$ ] L-shell range between 05:30 UT and 06:10 UT and cross the L-shell studied by Vallat et al. (2005). The average current density obtained here is 10 times higher than the one they obtained. It has been shown that the error can be different from one $\boldsymbol{J}_{\text {av }}$ component to another and in particular, that close to perigee, it will be more important on the parallel to the z-axis component. Therefore, we will now compare the azimuthal currents obtained for the 18 March 2002 and the 15 May 2009. For each event, a constant azimuthal current is obtained, but it is about equal to $-200 \mathrm{nA} \mathrm{m}^{-2}$ on the 15 May 2009, when it has been found at about $-20 \mathrm{nA} \mathrm{m}^{-2}$ on 18 March 2002. As a consequence, the results obtained for the first interval on the 15 May 2009 looks to be inaccurate.

Looking now at the second time interval, $\boldsymbol{J}_{\text {av }}$ decreases from $50 \mathrm{nA} \mathrm{m}^{-2}$ to $5 \mathrm{nA} \mathrm{m}^{-2}$ as well as $J_{\mathrm{av} \varphi}$, which appears to be a closer value to the one obtained for the 18 March 2002 event. All the same, from $L=6.3 R_{\mathrm{E}}$, the current defined in the model is westward and $J_{\mathrm{av} \varphi}$ has to be negative when the one calculated using the curlometer is positive. Such results indicate that, despite low $\operatorname{div} \boldsymbol{B}, \operatorname{div} \boldsymbol{B} / \boldsymbol{c u r l} \boldsymbol{B}$ and $\Delta b_{i j}$ values, $\boldsymbol{J}_{\text {av }}$ obtained using the curlometer for the 15 May 2009 perigee pass is not accurate and that the error induced by the curlometer technique, for this orbital configuration of the four spacecraft, is very high on each of the $\boldsymbol{J}_{\mathrm{av}}$ components. 


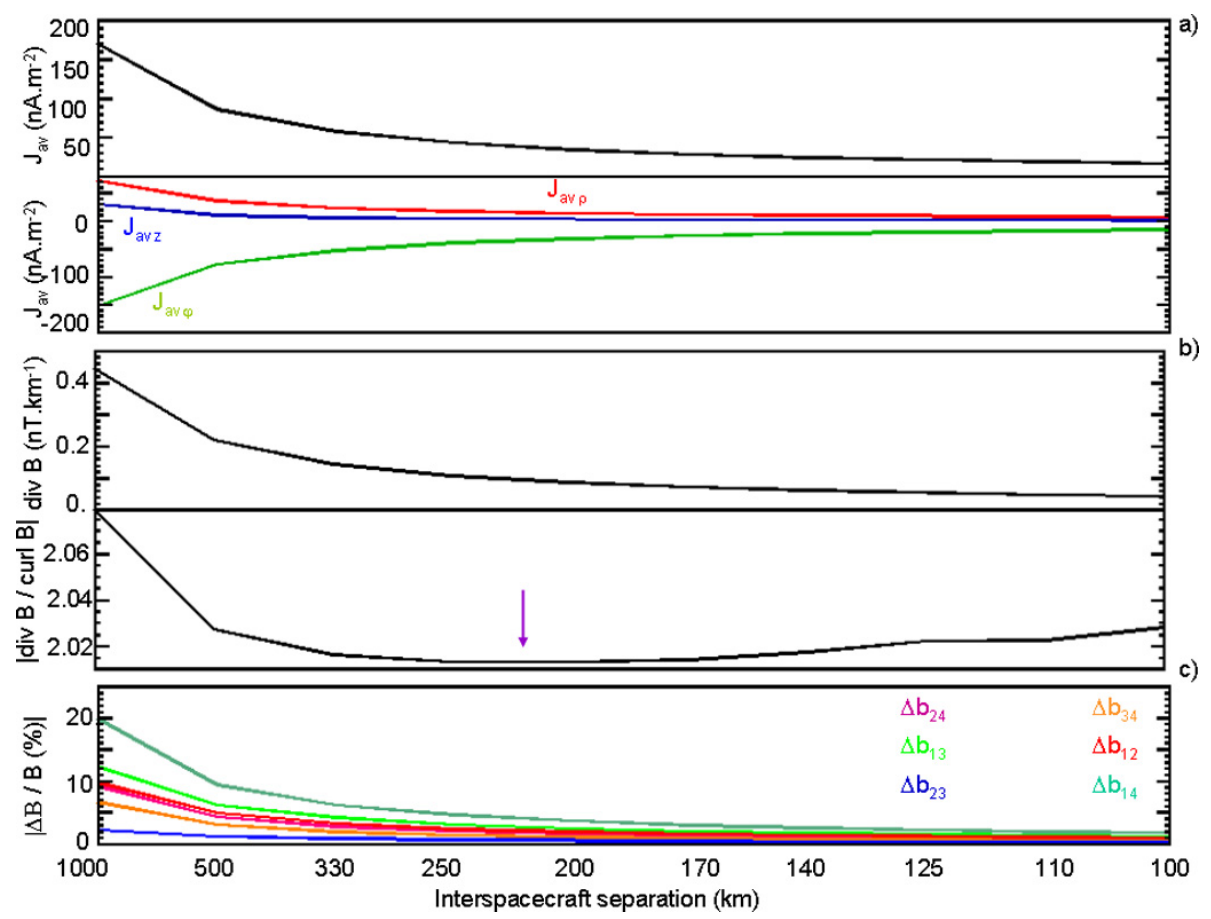

Fig. 10. $\boldsymbol{J}_{\mathrm{av}}$, its components (frame a), $\operatorname{div} \boldsymbol{B}$ (frame b), $\operatorname{div} \boldsymbol{B} / \boldsymbol{c u r l} \boldsymbol{B}$ (frame b), and $\Delta b_{i j}$ (frame c) versus the interspacecraft separation distance. The violet arrow is located at about $220 \mathrm{~km}$ separation distance. For this value, the best accuracy of the curlometer has been obtained.

\section{Calculation of the current density for smaller separations}

\subsection{Study of the curlometer results at the perigee pass for different separations}

Along the Cluster orbit, the shape of the tetrahedron changes: it is very elongated at perigee passes and flattened in the cusp crossings. To use the curlometer technique, the size of the tetrahedron has to be small enough so that the four satellites are located in the same current sheet and the magnetic field gradients are as linear as possible. At the same time, it has to also be big enough so as the magnetic field gradients are detectable from one satellite to another. For the 15 May 2009 ring current density calculations, the four satellites are located in the same current sheet, but the $\Delta B / B$ is quite high for the whole ring current region crossing. This result indicates that the distance between the satellites is too large to suppose that the magnetic field measured on board each satellite is very close to each other (linear gradient assumption). In the perspective of future missions, this addresses the question of whether the curlometer technique could be used for a smaller spacecraft separation. To answer this question, the first point is to determine for which interspacecraft separations accurate values of $\boldsymbol{J}_{\text {av }}$ are obtained. To do so, a given point of the 15 May 2009 orbit has been chosen to test the accuracy of the curlometer for different separations. As the method is inaccurate because of the rapid spatial evolution of the magnetic field, the perigee pass, where the magnetic field and its variations are the most important, has been chosen. Then, the curlometer has been used in the Tsyganenko magnetic field used in Sect. 4.2 for simulated tetrahedrons obtained by reducing its size by a factor between 1 and 10. Figure 10 presents $\boldsymbol{J}_{\mathrm{av}}$, its components (frame a), $\operatorname{div} \boldsymbol{B}$ (frame b), $\operatorname{div} \boldsymbol{B} / \boldsymbol{c u r l} \boldsymbol{B}$ (frame b), and $\Delta b_{i j}$ (frame c) versus the interspacecraft separation distance. Focusing on the $\operatorname{div} \boldsymbol{B} / \boldsymbol{c u r l} \boldsymbol{B}$ evolution, it is noticeable that it decreases until $220 \mathrm{~km}$ separation (violet arrow in Fig. 10), and then increases, showing that it exists a separation leading to the best accuracy of $\boldsymbol{J}_{\text {av }}$. Unfortunately, $\operatorname{div} \boldsymbol{B} / \operatorname{curl} \boldsymbol{B} \approx 2.01$ at its minimum, indicating not a good accuracy of the method at the perigee pass even for this separation.

\subsection{Current density for a $220 \mathrm{~km}$ separation}

It has been shown that $220 \mathrm{~km}$ separation gives the best accuracy of the curlometer for a perigee pass located at about $2 R_{\mathrm{E}}$, but that the error is quite important at this position. The tetrahedron at the perigee pass is very elongated. As a consequence, the error on the current density can be important at this position, but it could be much better in another position along the orbit. To conclude this study on the accuracy on the curlometer at low altitude, Fig. 11 presents the results obtained for a simulated Cluster orbit with a $220 \mathrm{~km}$ separation. The first frames in Fig. 11 present the geocentric distances 

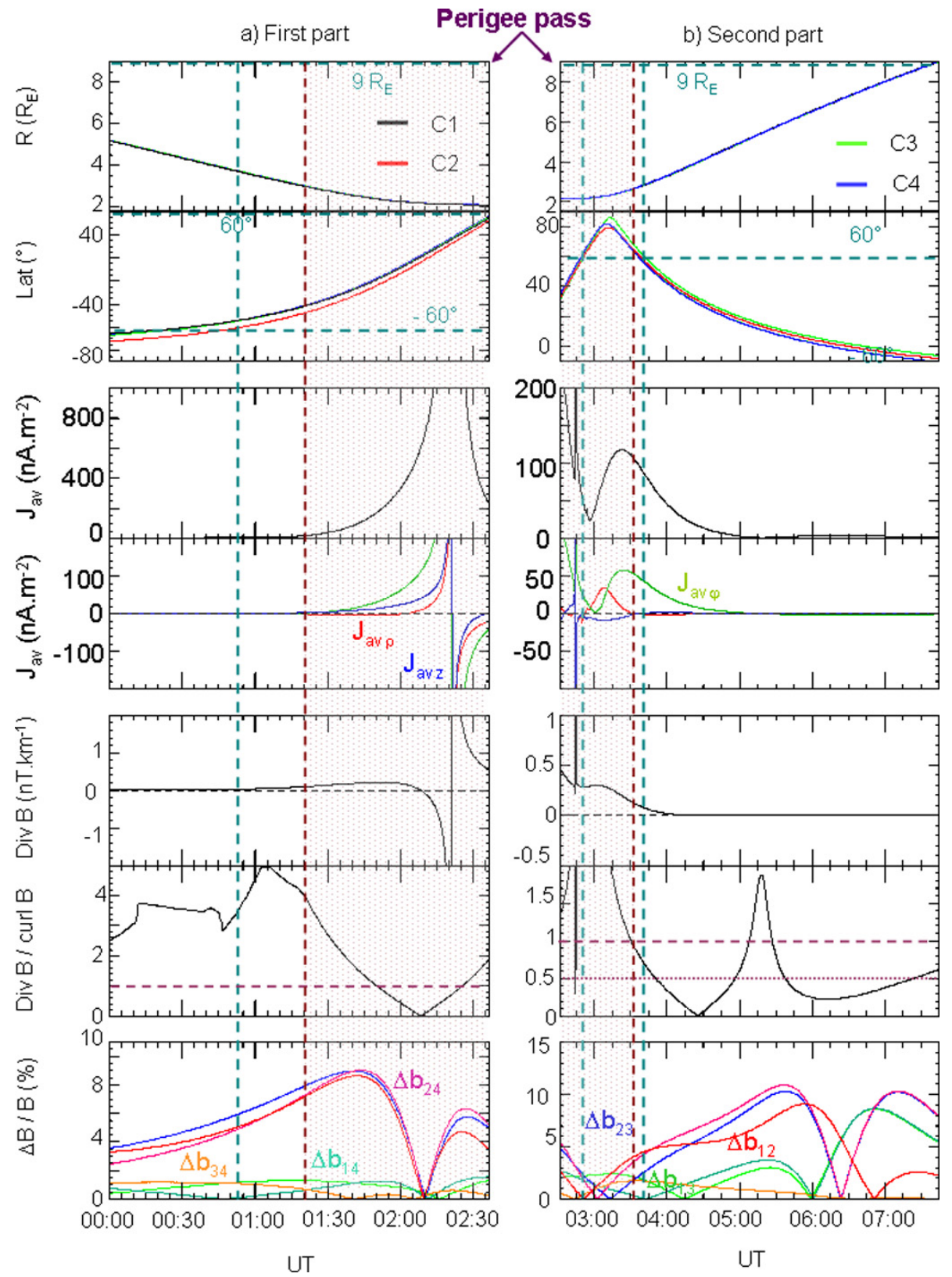

Fig. 11. Study of the accuracy of the curlometer technique for the Tsyganenko magnetic field calculated along a modelled orbit with $200 \mathrm{~km}$ separation.

(first panel) and the magnetic latitude (second panel) of the four satellites during the ring current region crossing. The perigee is located at $2 R_{\mathrm{E}}$ and is crossed at 02:35 UT. As it was necessary to use different scales before and after the perigee pass, the figure has been divided in two columns: before the perigee pass in the left part of the figure (annotated "a") and after the perigee pass in the right part of the figure (annotated "b"). It is clear from this plot that the satellites are very close to each other. As the ring current is typically observed in a $\left(2 R_{\mathrm{E}} ; 9 R_{\mathrm{E}}\right)$ geocentric distance range and $\pm 60^{\circ}$ magnetic latitude (Vallat et al., 2005), horizontal blue dashed lines (first frames of Fig. 11a and b) have been located at $9 R_{\mathrm{E}}$ (first panels) and at $\pm 60^{\circ}$ magnetic latitude (second panels). The vertical blue dashed lines indicate the boundaries of the ring current: the satellites are located inside the ring current in a [00:54 UT; 02:40 UT] and in a [03:40 UT; 07:15 UT] time range. The magnetic field along the orbit has been calculated using a Tsyganenko model (Tsyganenko and Stern, 1996) and then used to calculate the current density from the curlometer. The third and the fourth frames of Fig. 11 present the results obtained from the calculations $\left(\boldsymbol{J}_{\mathrm{av}}\right.$, its components in the third, $\operatorname{div} \boldsymbol{B}$ and $\operatorname{div} \boldsymbol{B} / \boldsymbol{c u r l} \boldsymbol{B}$ in the fourth one). 
The last frame presents the time evolution of $\Delta b_{i j}$ which is never higher than $12 \%$. As in Figs. 6, 7 and 9, the brown dashed lines are located where $|\operatorname{div} \boldsymbol{B}|=0.1 \mathrm{nT} \mathrm{km}^{-1}$ and the brown dotted areas indicate when $\operatorname{div} B>0.1$. In panel (a), the satellites are located in the ring current region and $|\operatorname{div} \boldsymbol{B}|<0.1$ between 00:54 UT and 01:21 UT. Unfortunately, during this time range, $\operatorname{div} \boldsymbol{B} / \operatorname{curl} \boldsymbol{B}>3.6$. As a consequence, the accuracy of the curlometer technique is small for this part of the orbit. Focusing now on the second part of the orbit (figure b), the satellites are located in the ring current region and $|\operatorname{div} \boldsymbol{B}|<0.1$ from 03:40 UT. $\operatorname{div} \boldsymbol{B} /$ curl $\boldsymbol{B}<0.5$ between 04:47 UT and 05:00 UT, and between 05:40 UT and 07:15 UT. As a consequence, the curlometer parameters indicate that the calculation may be accurate for such an orbit in an [ $3 R_{\mathrm{E}} ; 5 R_{\mathrm{E}}$ ] and [ $6 R_{\mathrm{E}} ; 8.3 R_{\mathrm{E}}$ ] $\mathrm{L}$-shell range with a $220 \mathrm{~km}$ separation.

\section{Discussion and conclusion}

In this paper, the curlometer technique has been used to calculate the current density much deeper inside the inner magnetosphere than ever before. In the first instance, the calculation was performed for a medium inter-spacecraft separation of the 4 Cluster satellites $(1000 \mathrm{~km})$ using the magnetic field time series measured by the FGM instruments during the 15 May 2009 ring current region crossing and using also three modelled magnetic field time series calculated along the same orbit (from an empirical model, a dipolar model and by subtracting the dipolar from the empirical magnetic field values). The results can be summarized as follows:

1. The Maxwell-Thomson law $(\operatorname{div} \boldsymbol{B}=0)$ is violated for each of the four magnetic field inputs, and $\operatorname{div} \boldsymbol{B} / \operatorname{curl} \boldsymbol{B}$ and $\Delta B / B$ are quite high for at least part of each time series. These results come from the rapid change of the magnetic field magnitude associated with the increased elongation of the tetrahedron at the perigee pass.

2. A non-zero current density has been obtained for the dipolar model showing the inadequacy of the method for this orbital configuration.

3. Regarding the last magnetic field model ( $\boldsymbol{B}_{\text {Tsyganenko }}-$ $\left.\boldsymbol{B}_{\text {dipole }}\right), \operatorname{div} \boldsymbol{B}, \operatorname{div} \boldsymbol{B} / \operatorname{curl} \boldsymbol{B}$ and $\Delta B / B$ evolutions seem to indicate a good accuracy of the method when the azimuthal current density is higher than the past results.

This study shows that the current density cannot be calculated using the curlometer technique for Cluster orbits with low altitude perigee passes, where the inter-spacecraft separation becomes too large. Moreover, we find that $\operatorname{div} \boldsymbol{B}, \operatorname{div} \boldsymbol{B} / \operatorname{curl} \boldsymbol{B}$ and $\Delta B / B$ characteristics are not enough to evaluate the accuracy of the method: $\operatorname{div} \boldsymbol{B} \approx 0$, $\operatorname{div} B /$ curl $B \ll 1$ and $\Delta B / B \ll 1$ are necessary but not sufficient conditions. The next step was to find a new condition that had to be sufficient to test the accuracy of the curlometer. Furthermore, it was shown that the error could vary from a current density to another. In particular, as the tetrahedron becomes more and more elongated when travelling to the perigee, the error on the z-component is higher than for the $\mathrm{x}$ - and the $\mathrm{y}$-components. Thus, it was found useful to define a test of the accuracy for each component of the current density calculated using the curlometer.

As a second step, the curlometer has been used at the perigee pass for different inter-spacecraft separations. This study shows that a separation that leads to the best accuracy of the method could be defined. It also shows that the current density cannot be calculated using the curlometer technique for perigee passes located as low as $2 R_{\mathrm{E}}$. Nevertheless, the calculation may be accurate within $\left[3 R_{\mathrm{E}} ; 5 R_{\mathrm{E}}\right]$ and [6 $R_{\mathrm{E}} ; 8.3 R_{\mathrm{E}}$ ] L-shell ranges, which are deeper inside the inner magnetosphere than what has been reported in the past.

Other methods are under development, which may work along such an orbit. Moreover, the MMS (Multi scale Magnetospheric Satellites) mission is composed of four satellites, which will have a separation of 10 to $200 \mathrm{~km}$ and a perigee located at $1.2 R_{\mathrm{E}}$ geocentric distance. Those satellites, to be launched in 2014, will have a separation distance very close to the one leading to the best accuracy of the method. It may bring new information about the current density in the inner magnetosphere and the parameters we have to use to test the accuracy of the curlometer.

Acknowledgements. S.G. acknowledges financial support from the Centre National d'Etudes Spatiales (CNES). We would like to thank the WEC, JSOC and ESOC teams for continuous support of Cluster operations. The authors thank the FGM and the CIS teams for preparing the data used in this paper, and the CAA for making the Cluster data available to the community. We also acknowledge the WDC for providing the magnetic indices. Data analysis was done with the QSAS science analysis system provided by the United Kingdom Cluster Science Centre (Imperial College London and Queen Mary, University of London) supported by STFC and the CL analysis software developed at IRAP.

Guest Editor M. Taylor thanks two anonymous referees for their help in evaluating this paper.

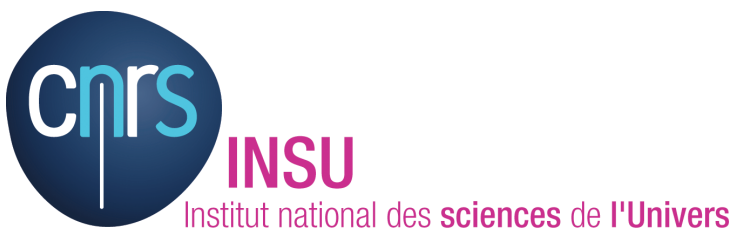

The publication of this article is financed by CNRS-INSU. 


\section{References}

Alfvén, H.: Some properties of magnetospheric neutral surfaces, J. Geophys. Res., 73, 4379-4381, 1968.

Balogh, A., Dunlop, M. W., Cowley, S. W. H., Soothwood, D. J., Thomlinson, J. G., Glassmeier, K. H., Musmann, G., Luhr, H., Buchert, S., Acuna, M. H., Fairfield, D. H., Slavin, J. A., Riedler, W., Schwingenschuh, K., and Kivelson, M. G.: The Cluster magnetic field investigation, Space Sci. Rev., 79, 65-91, 1997.

Balogh, A., Carr, C. M., Acuña, M. H., Dunlop, M. W., Beek, T. J., Brown, P., Fornacon, K.-H., Georgescu, E., Glassmeier, K.H., Harris, J., Musmann, G., Oddy, T., and Schwingenschuh, K.: The Cluster Magnetic Field Investigation: overview of in-flight performance and initial results, Ann. Geophys., 19, 1207-1217, doi:10.5194/angeo-19-1207-2001, 2001.

Buzulukova, N. Y., Galperin, Y. I., Kovrazhkin, R. A., Glazunov, A. L., Vladimirova, G. A., Stenuit, H., Sauvaud, J. A., and Delcourt, D. C.: Two types of ion spectral gaps in the quiet inner magnetosphere: Interball-2 observations and modeling, Ann. Geophys., 20, 349-364, doi:10.5194/angeo-20-349-2002, 2002.

Chanteur, C.: Spatial interpolation for four spacecraft: theory, in Analysis Methods for Multi-Spacecraft data, ISSI Sci. Rep. SR001, 323-448, 1998.

Chanteur, C. and Mottez, F.: Geometricl tools for Cluster data analysis, in: Proc. International Conf, "Spatio-temporal Analysis plasma turbulence (START), Aussois, 31 January-5 February 1993, ESA WPP-047, pp. 341-344, European Space Agency, Paris, France, 1993.

Chen, M. W., Lui, S., Schulz, M., Roeder, J. L., and Lyons, L. R.: Magnetically self-consistent ring current simulations during the 19 October 1998 storm, J. Geophys. Res., 111, A11S15, doi:10.1029/2006JA011620, 2006.

Daglis, I. A., Sarris, E. T., and Wilken, B.: AMPTS/CCE CHEM observations of the ion population at geosynchronous altitude, Ann. Geophys., 11, 685-696, 1993, http://www.ann-geophys.net/11/685/1993/.

Dunlop, W. M., Southwood, D. J., Glassmeier, K.-H., and Neubauer, F. M.: Analysis of multipoint magnetometer data, Adv. Space Res., 8, 9-10, 1988.

Daglis, I. A., Thorne, R. M., Baumjohann, W., and Orsini, S.: The terrestrial ring current: origin, formation, and decay, Rev. of Geophys., 37, 407-438, 1999.

Dandouras, I., Cao, J., and Vallat, C.: Energetic ion dynamics of the inner magnetosphere revealed in coordinated ClusterDouble Star observations, J. Geophys. Res., 114, A01S90, doi:10.1029/2007JA012757, 2009.

Dunlop, M. W. and Balogh, A.: On the analysis and the interpretation of the four spacecraft magnetic field measurements in term of small scale plasma processes, in Spatio-Temporal Analysis for Resolving Plasma Turbulence (START), Eur. Space Agency, WPP, ESA WPP-047, p. 223, 1993.

Dunlop, M. W., Balogh, A., Glassmeier, K.-H., and Robert, P.: Four-point Cluster application of magnetic field analysis tools: the curlometer, J. Geophys. Res., 107, 1384-1398, 2002.

Ejiri, M.: Trajectory traces of charged particles in the magnetosphere, J. Geophys. Res., 83, 4798-4810, doi:10.1029/JA083iA10p04798, 1978.

Escoubet, C. P., Fehringer, M., and Goldstein, M.: Introduction "The Cluster mission", Ann. Geophys., 19, 1197-1200, doi:10.5194/angeo-19-1197-2001, 2001.
Ganushkina, N. Yu., Dandouras, I., Shprits, Y. Y., and Cao, J.: Locations of Boundaries of Outer and Inner Radiation Belts as Observed by Cluster and Double Star, J. Geophys. Res., 116, A09234, doi:10.1029/2010JA016376, 2011.

Gloeckler, G. B., Wilkinen, W., Stüdemann, F., Ipavich, F. M., Hovestadt, D., Hamilton, D. C., and Kremser, G.: First composition measurement of the bulk of the storm-time ring current (1 to $300 \mathrm{keV} / \mathrm{e}$ ) AMPTE/CCE, Geophys. Res. Lett., 12, 325-328, 1985.

Hamilton, D. C., Gloeckler, G., Ipavitch, F. M., Stüdemann, W., Wilken, B., and Kremser, G.: Ring current development during the great geomagnetic storm of February 1986, J. Geophys. Res., 93, 14343-14355, 1988.

Jordanova, V. K.: New Insights on Geomagnetic Storms from Model Simulations Using Multi-Spacecraft Data, Space Sci. Rev., 107, 157-165, doi:10.1023/A:1025575807139, 2003.

Jordanova, V. K., Miyoshi, Y. S., Zaharia, S., Thomsen, M. F., Reeves, G. D., Evans, D. S., Mouikis, C. G., and Fennell, J. F.: Kinetic simulations of ring current evolution during the Geospace Environment Modeling challenge events, J. Geophys. Res., 111, A11S10, doi:10.1029/2006JA011644, 2006.

Jorgensen, A. M., Spence, H. E., Hughes, W. J., and Singer, H. J.: A statistical study of the global structure of the ring current, J. Geophys. Res., 109, A12204, doi:10.1029/2003JA010090, 2004.

Krimigis, S. M., Gloeckler, G., McEntire, R. W., Potemra, T. A., Scarf, F. L., and Shelley, E. G.: Magnetic storm of September 4, 1984: A synthesis of ring current spectra and energy densities measured with AMPTE/CCE, Geophys. Res. Lett., 12, 329-332, 1985.

Le, G., Russell, C. T., and Takahashi, K.: Morphology of the ring current derived from magnetic field observations, Ann. Geophys., 22, 1267-1295, doi:10.5194/angeo-22-1267-2004, 2004.

Lui, A. T. Y. and Hamilton, D. C.: Radial profiles of quiet time magnetospheric parameters, J. Geophys. Res., 97, 19325-19332, 1992.

Lui, A. T. Y., Mc Entire, R. W., and Krimigis, S. M.: Evolution of the ring current during two geomagnetic storm, J. Geophys. Res., 92, 7459-7470, 1987.

McIlwain, C.: Plasmaconvection in the vicinity of the geosynchronous orbit, in Earth's Magnetospheric Processes, McCormac, B., p. 268, D. Reidel Pub. Comp., 1972.

Rème, H., Aoustin, C., Bosqued, J. M., Dandouras, I., Lavraud, B., Sauvaud, J. A., Barthe, A., Bouyssou, J., Camus, Th., CoeurJoly, O., Cros, A., Cuvilo, J., Ducay, F., Garbarowitz, Y., Medale, J. L., Penou, E., Perrier, H., Romefort, D., Rouzaud, J., Vallat, C., Alcaydé, D., Jacquey, C., Mazelle, C., d'Uston, C., Möbius, E., Kistler, L. M., Crocker, K., Granoff, M., Mouikis, C., Popecki, M., Vosbury, M., Klecker, B., Hovestadt, D., Kucharek, H., Kuenneth, E., Paschmann, G., Scholer, M., Sckopke, N., Seidenschwang, E., Carlson, C. W., Curtis, D. W., Ingraham, C., Lin, R. P., McFadden, J. P., Parks, G. K., Phan, T., Formisano, V., Amata, E., Bavassano-Cattaneo, M. B., Baldetti, P., Bruno, R., Chionchio, G., Di Lellis, A., Marcucci, M. F., Pallocchia, G., Korth, A., Daly, P. W., Graeve, B., Rosenbauer, H., Vasyliunas, V., McCarthy, M., Wilber, M., Eliasson, L., Lundin, R., Olsen, S., Shelley, E. G., Fuselier, S., Ghielmetti, A. G., Lennartsson, W., Escoubet, C. P., Balsiger, H., Friedel, R., Cao, J.-B., Kovrazhkin, R. A., Papamastorakis, I., Pellat, R., Scudder, J., and Sonnerup, B.: First multispacecraft ion measurements in and near the Earth's 
magnetosphere with the identical Cluster ion spectrometry (CIS) experiment, Ann. Geophys., 19, 1303-1354, doi:10.5194/angeo19-1303-2001, 2001.

Robert, P. and Roux, A.: Dependance of the shape of the tetrahedron on the accuracy of the estimate of the current density, in Spatiotemporal Analysis for Resolving Plasma Turbulence (START), Eur. Space Agency, WPP, ESA WPP-047, 289-193, 1993.

Robert, P., Dunlop, M. W., Roux, A., and Chanteur, G.: Accuracy of current density determination, in Analysis Methods for MultiSpacecraft data, ISSI Sci. Rep. SR-001, 395-418, 1998a.

Robert, P., Roux, A., Harvey, C. C., Dunlop, M. W., Daly, P. W., and Glassmeier, K.-H.: Tetrahedron geometric factors, in: Analysis Methods for Multi-Spacecraft data, ISSI Sci. Rep. SR-001, 323448, 1998b.

Roelof, E. C., C:son Brandt, P., and Mitchell, D. G.: Derivation of currents and diamagnetic effects from global plasma pressure distributions obtained by IMAGE/HENA, Adv. Space Res., 33, 747-751, 2004.

Sauvaud, J.-A., Barthe, H., Aoustin, C., Thocaven, J. J., Penou, E., Rouzaud, J., Kovrazhkin, R. A., Afanasiev, K. G., and Ivanchenkova, I. Yu.: Measurement of the suprathermal plasma by ION spectrometric complex on the Interball-2 satellite (Auroral probe), Cosmic Research, 36, 59-68, 1998a.

Sauvaud, J. A., Barthe, H., Aoustin, C., Thocaven, J. J., Rouzaud, J., Penou, E., Popescu, D., Kovrazhkin, R. A., and Afanasiev, K. G.: The ion experiment onboard the Interball-Aurora satellite; initial results on velocity-dispersed structures in the cleft and inside the auroral oval, Ann. Geophys., 16, 1056-1069, doi:10.1007/s00585-998-1056-z, 1998 b.

Singer, S. F.: A new model of magnetic storms and aurorae, Eos Trans. AGU, 38, 175-190, 1957.
Shirai, H., Maezawa, K., Fujimoto, M., Mukai, T., Saito, Y., and Kaya, N.: Monoenergetic ion drop-off in the inner magnetosphere, J. Geophys. Res., 102, 19873-19881, doi:10.1029/97JA01150, 1997.

Smith, P. H. and Hoffman, R. A.: Ring current particle distributions during the magnetic storms of December 16-19, 1971, J. Geophys. Res., 78, 4731-4737, 1973.

Stern, D. P.: The motion of a proton in the equatorial magnetosphere, J. Geophys. Res., 80, 595-599, 1975.

Tsyganenko, N. A. and Stern, D. P.: Modeling the global magnetic field of the large-scale Birkeland current systems, J. Geophys. Res., 101, 27187-27198, 1996.

Vallat, C., Dandouras, I., Dunlop, M., Balogh, A., Lucek, E., Parks, G. K., Wilber, M., Roelof, E. C., Chanteur, G., and Rème, H.: First current density measurements in the ring current region using simultaneous multi-spacecraft CLUSTER-FGM data, Ann. Geophys., 23, 1849-1865, doi:10.5194/angeo-23-18492005, 2005.

Vallat, C., Ganushkina, N., Dandouras, I., Escoubet, C. P., Taylor, M. G. G. T., Laakso, H., Masson, A., Sauvaud, J.-A., Rème, H., and Daly, P.: Ion multi-nose structures observed by Cluster in the inner Magnetosphere, Ann. Geophys., 25, 171-190, doi:10.5194/angeo-25-171-2007, 2007.

Volland, H.: A semi empirical model of large-scale magnetospheric electric fields, J. Geophys. Res., 78, 171, doi:10.1029/JA078i001p00171, 1973.

Zaharia, S., Jordanova, V. K., Thomsen, M. F., and Reeves, G. D.: Self-consistent modeling of magnetic fields and plasmas in the inner magnetosphere: Application to a geomagnetic storm, J. Geophys. Res., 111, A11S14, doi:10.1029/2006JA011619, 2006. 